\title{
Analytical design of maximally flat FIR fractional Hilbert transformers
}

\author{
Soo-Chang Pei*, Peng-Hua Wang \\ Department of Electrical Engineering, National Taiwan University, Taipei, Taiwan, ROC
}

Received 29 November 1999; received in revised form 23 May 2000; accepted 8 October 2000

\begin{abstract}
Recently, the classical Hilbert transformer is generalized into the fractional Hilbert transformer which could be implemented optically. This modification of the Hilbert transform adds an additional degree of freedom on the Hilbert transformer and improves the performance of the transform. In this paper, the design of FIR filters fractional Hilbert transformer is proposed. The FIR filters are designed in the maximally flat sense. The impulse responses of the filters are uniquely solved and expressed in simple analytic forms. The impulse responses can be exactly expressed as fixed point binary values. The resulting frequency responses approximate the ideal one very well in the middle-frequency band. Efficient hardware realization structures are obtained based on the symmetric properties of the impulse responses. Several design examples with various transform parameters and various filter orders are presented. Some examples of 1-D/2-D edge detection are given. The examples show that the proposed FIR filter can enhance the selected edges very efficiently. (C) 2001 Elsevier Science B.V. All rights reserved.
\end{abstract}

Keywords: Hilbert transformer; Fractional Hilbert transformer; Maximal flatness; FIR filter

\section{Introduction}

Recently, the classical Hilbert transformers (HTs) are generalized into the fractional Hilbert transformers (FHTs) which could be implemented optically [8]. The modification of the Hilbert transform adds additional degrees of freedom on the Hilbert transformer and improves the performance of the transform on edge detection. Two alternative definitions were proposed in [8]. The first definition modifies the spatial frequency response of the

\footnotetext{
* Corresponding author. Tel.: + 886-2-2363-5251; fax: 886-223671909.

E-mail address: pei@cc.ee.ntu.edu.tw (S.-C. Pei).
}

classical Hilbert transform with a fractional parameter. The second definition makes use of the classical Hilbert transform as well as the fractional Fourier transform to fractionalize the Hilbert transform. The fractional parameter is controlled by the fractional Fourier transform in the second definition. However, the Hilbert transform is a special case in both definitions.

For digital computation and implementation, the discrete versions of the two definitions of the fractional Hilbert transform were proposed and developed in [13]. The authors derived a frequency mask by which the first definition of fractional Hilbert transform can be computed in the discretetime frequency domain. In the second definition, the fractional Fourier transform is replaced by the 
discrete fractional Fourier transform [14]. Therefore, both of the definitions of the fractional Hilbert transformer can be computed in the discrete-time domain.

The design of digital filters which perform the Hilbert transform has been widely investigated. The ideal impulse response of the Hilbert transformer $[11,15]$ is given by

$h_{\mathrm{ht}}(n)= \begin{cases}\frac{2 \sin ^{2}(n \pi / 2)}{n \pi}, & n \neq 0, \\ 0, & n=0 .\end{cases}$

The design of the optimal FIR linear phase digital filter in the minimax sense has been often used in practice $[9,16]$. The performance of the FIR Hilbert transformer can be improved by multiple use of identical FIR subfilters [18]. In [1,7], the impulse responses of the FIR Hilbert transformer are analytically solved in the maximally flat (MF) sense. A realization scheme based on decomposing the transfer function of Hilbert transformer into allpass subfilters was proposed in [5].

For image processing, the Hilbert transform is used especially for edge detection [6]. The fractional Hilbert transform can be used for a finer detection of the edge. A detailed analysis about the fractional Hilbert transform on the rectangle function was proposed [2]. The authors explained how the one-dimensional edges are selectively emphasized by fractional Hilbert transform.

In this paper, a design of the maximally flat FIR fractional Hilbert transformer is proposed. We want to design a digital FIR filter to perform the fractional Hilbert transform. The first definition of the fraction Hilbert transformer in [8] is taken as our target frequency response since this definition does not involve the time-variant operation of fractional Fourier transform and can be implemented by a digital filter. This target frequency response in the discrete-time frequency domain can be expressed by

$H_{\text {fht }}(\omega)= \begin{cases}\mathrm{e}^{-\mathrm{j} \alpha \pi / 2} & \text { for } 0<\omega<\pi, \\ \mathrm{e}^{\mathrm{j} \alpha \pi / 2} & \text { for }-\pi<\omega<0,\end{cases}$

where $\alpha$ is the fractional parameter. In order to design a causal FIR filter, the ideal frequency response expressed in Eq. (1) must be modified be- cause the response lacks of some delays and cannot be realized in practice. For the $N$ th-order FIR filter to be designed, we put an additional delay term $\mathrm{e}^{-\mathrm{j} \omega \omega / 2}$ into Eq. (1) and obtain the desired frequency response as

$H_{\mathrm{d}}\left(\mathrm{e}^{\mathrm{j} \omega}\right)= \begin{cases}\mathrm{e}^{-\mathrm{j}(\alpha \pi / 2+N \omega / 2)} & \text { for } 0<\omega<\pi, \\ \mathrm{e}^{\mathrm{j}(\alpha \pi / 2-N \omega / 2)} & \text { for }-\pi<\omega<0 .\end{cases}$

In the odd order case, Pei and Wang have obtained an analytical expression of the impulse response [12]. An efficient hardware realization structure based on the symmetry of the filter coefficients was proposed. In this paper, we will make an extension of this previous work. In Section 2, we formulate the problem for the design of the maximally flat FIR fractional Hilbert transformers. The analytical impulse response of odd-order fractional Hilbert transformer is developed and given in this section. In Section 3, we solve the even-order maximally flat FIR fractional Hilbert transformer. Analytical expressions of the impulse responses are obtained. Several design examples are given in Section 4, including a comparison between the odd-order filters and the even-order ones for edge detection applications. Finally, we give a conclusion in Section 5 .

\section{Design of odd-order FIR FHTs}

The transfer function of a complex coefficient FIR digital filter is characterized by

$H(z)=\sum_{n=0}^{N} h(n) z^{-n}$,

where $N$ is the filter order, and the filter coefficients $h(n)$ generally complex-valued. The frequency response of this FIR filter is obtained by evaluating $H(z)$ along the unit circle and expressed by

$H\left(\mathrm{e}^{\mathrm{j} \omega}\right)=\sum_{n=0}^{N} h(n) \mathrm{e}^{-\mathrm{j} n \omega}$.

We want to find $h(n)$ such that $H\left(\mathrm{e}^{\mathrm{j} \omega}\right)$ is approximated to the desired response $H_{\mathrm{d}}\left(\mathrm{e}^{\mathrm{j} \omega}\right)$.

To design a FIR filter in the maximally flat sense, we equate the derivatives of the desired frequency 
response in Eq. (2) with the derivatives of $H\left(\mathrm{e}^{\mathrm{j} \omega}\right)$ up to a suitable $K$ th order, i.e.,

$$
\begin{gathered}
\sum_{n=0}^{N} h(n) n^{k} \mathrm{e}^{-\mathrm{j} n \omega}=\left(\frac{N}{2}\right)^{k} \mathrm{e}^{-\mathrm{j}(\alpha \pi / 2+N \omega / 2)} \\
\text { for } 0<\omega<\pi
\end{gathered}
$$

and

$$
\begin{gathered}
\sum_{n=0}^{N} h(n) n^{k} \mathrm{e}^{-\mathrm{j} n \omega}=\left(\frac{N}{2}\right)^{k} \mathrm{e}^{\mathrm{j}(\alpha \pi / 2-N \omega / 2)} \\
\text { for }-\pi<\omega<0
\end{gathered}
$$

for $k=0,1, \ldots, K$ where $K$ is determined by the filter order $N$. In fact, $K=(N-1) / 2$.

For the maximally flat (MF) design, the next step is to choose the frequencies at which the MF conditions expressed by Eqs. (5) and (6) are satisfied. We choose the two MF frequencies of $\pm \pi / 2$ to approximate the desired frequency response. Substituting Eqs. (5) and (6) with $\omega=\pi / 2$ and $-\pi / 2$, respectively, these equations become

$$
\begin{aligned}
& \sum_{n=0}^{N} h(n) n^{k} \mathrm{e}^{-\mathrm{j} n \pi / 2}=\left(\frac{N}{2}\right)^{k} \mathrm{e}^{-\mathrm{j} \phi}, \\
& \sum_{n=0}^{N} h(n) n^{k} \mathrm{e}^{\mathrm{j} n \pi / 2}=\left(\frac{N}{2}\right)^{k} \mathrm{e}^{\mathrm{j} \phi},
\end{aligned}
$$

where $\phi=\alpha \pi / 2+N \pi / 4$ for short.

In order to determine the largest allowable $k$ in the above equations, the number of these equations is examined. For the $k$ th derivative, two MF conditions must be satisfied since the MF frequencies are specified to be $\pi / 2$ and $-\pi / 2$. That is, there are two equations constructed for each choice of $k$. To solve the linear equations with $N+1$ unknowns expressed by Eqs. (7) and (8), $k$ is equal to $(N-1) / 2$ at most. If the largest $k$, i.e., $K$, is equal to $(N-1) / 2$, there are $N+1$ linear equations in Eqs. (7) and (8), the impulse response of the MF FHT can be solved exactly.

To solve these equations analytically, they are simplified further. For each $k$, by adding Eqs. (7) and (8) and by subtracting Eqs. (7) from Eq. (8), we deduce

$$
\begin{aligned}
& \sum_{n=0}^{N} h(n) n^{k} \cos \left(\frac{n \pi}{2}\right)=\left(\frac{N}{2}\right)^{k} \cos \phi, \\
& \sum_{n=0}^{N} h(n) n^{k} \sin \left(\frac{n \pi}{2}\right)=\left(\frac{N}{2}\right)^{k} \sin \phi
\end{aligned}
$$

for $k=0,1, \ldots,(N-1) / 2$. Since $\cos (n \pi / 2)=0$ and $\sin (n \pi / 2)=0$ for odd $n$ and even $n$, respectively, the above equations are actually in the forms

$$
\begin{aligned}
& \sum_{n=0}^{(N-1) / 2} h(2 n)(-1)^{n}(2 n)^{k}=\left(\frac{N}{2}\right)^{k} \cos \phi, \\
& \sum_{n=0}^{(N-1) / 2} h(2 n+1)(-1)^{n}(2 n+1)^{k}=\left(\frac{N}{2}\right)^{k} \sin \phi
\end{aligned}
$$

for $\quad k=0,1, \ldots,(N-1) / 2$. Denote $\tilde{h}(2 n)=$ $h(2 n)(-1)^{n} / \cos \phi$ and $\tilde{h}(2 n+1)=h(2 n+1)(-1)^{n} /$ $\sin \phi$ for $n=0,1, \ldots,(N-1) / 2$. The matrix forms of Eqs. (11) and (12) are expressed as

$$
\begin{aligned}
\left(\begin{array}{ccccc}
1 & 1 & 1 & \ldots & 1 \\
0 & 2 & 4 & \cdots & (N-1) \\
0 & 2^{2} & 4^{2} & \cdots & (N-1)^{2} \\
\vdots & \vdots & \vdots & \ddots & \vdots \\
0 & 2^{(N-1) / 2} & 4^{(N-1) / 2} & \cdots & (N-1)^{(N-1) / 2}
\end{array}\right)\left(\begin{array}{c}
\tilde{h}(0) \\
\tilde{h}(2) \\
\tilde{h}(4) \\
\vdots \\
\tilde{h}(N-1)
\end{array}\right) \\
=\left(\begin{array}{c}
1 \\
N / 2 \\
(N / 2)^{2} \\
\vdots \\
(N / 2)^{(N-1) / 2}
\end{array}\right)
\end{aligned}
$$

and

$$
\begin{aligned}
\left(\begin{array}{ccccc}
1 & 1 & 1 & \cdots & 1 \\
1 & 3 & 5 & \cdots & N \\
1 & 3^{2} & 5^{2} & \cdots & N^{2} \\
\vdots & \vdots & \vdots & \ddots & \vdots \\
1 & 3^{(N-1) / 2} & 5^{(N-1) / 2} & \cdots & N^{(N-1) / 2}
\end{array}\right)\left(\begin{array}{c}
\tilde{h}(1) \\
\tilde{h}(3) \\
\tilde{h}(5) \\
\vdots \\
\tilde{h}(N)
\end{array}\right) \\
=\left(\begin{array}{c}
1 \\
N / 2 \\
(N / 2)^{2} \\
\vdots \\
(N / 2)^{(N-1) / 2}
\end{array}\right),
\end{aligned}
$$

respectively. Note that the coefficient matrices of the above equations are Vandermonde matrices [4]. 
Since both Eqs. (11) and (12) describe $(N+1) / 2$ equations in $(N+1) / 2$ unknowns, these two sets of equations can be solved directly. Accordingly, the coefficients of the even numbered impulses and those of the odd numbered ones are solved separately. Carrying out Cramer's rule [10] and using the involved Vandermonde determinants, by some algebraic manipulations, the solutions are obtained explicitly by

$h(2 n)=\frac{k_{\mathrm{e}} \cos \phi}{(n-N / 4)((N-1) / 2-n) ! n !}$,

$h(2 n+1)=\frac{k_{\mathrm{o}} \sin \phi}{(n-(N-2) / 4)((N-1) / 2-n) ! n !}$,

where

$k_{\mathrm{e}}=\prod_{i=0}^{(N-1) / 2}\left(i-\frac{N}{4}\right)$

$k_{\mathrm{o}}=\prod_{i=0}^{(N-1) / 2}\left(i-\frac{N-2}{4}\right)$.

The impulse response exhibits fine symmetry. Let the normalized impulse responses be $\hat{h}(2 n)=h(2 n) /$ $\cos \phi$ and $\hat{h}(2 n+1)=h(2 n+1) / \sin \phi$. Then the transfer function expressed by Eq. (3) is represented as

$H(z)=\cos \phi H_{\mathrm{e}}(z)+\sin \phi z^{-1} H_{\mathrm{o}}(z)$,

where

$H_{\mathrm{e}}(z)=\sum_{n=0}^{(N-1) / 2} \hat{h}(2 n) z^{-2 n}$

$H_{\mathrm{o}}(z)=\sum_{n=0}^{(N-1) / 2} \hat{h}(2 n+1) z^{-2 n}$

The first property of the impulse response is that the factor $k_{\mathrm{o}}$ is equal to $k_{\mathrm{e}}$ without regard to their signum. This fact is obtained by changing the multiplication order in Eq. (16) and explicitly expressed as

$k_{\mathrm{o}}=(-1)^{(N+1) / 2} k_{\mathrm{e}}$.

By means of the above equality, we deduce that

$\hat{h}(N-2 n)=(-1)^{(N-1) / 2} \hat{h}(2 n)$ for $n=0,1, \ldots,(N-1) / 2$. Note that $(N-2 n)=$ $[2((N-1) / 2-n)+1]$, i.e., $\hat{h}(N-2 n)$ is an odd numbered coefficient while $\hat{h}(2 n)$ is an even numbered one.

The key equation (21) implies the important property of $H_{\mathrm{o}}(z)$ being the mirror-image polynomial about $H_{\mathrm{e}}(z)$, i.e.,

$H_{\mathrm{o}}(z)=(-1)^{(N-1) / 2} z^{-(N-1)} H_{\mathrm{e}}\left(z^{-1}\right)$.

Based on this symmetry property, an efficient realization structure is designed. We begin with the fact that any FIR filter can be expressed as a sum of a symmetric and an antisymmetric FIR filter. Accordingly $H_{\mathrm{e}}(z)$ is decomposed as

$H_{\mathrm{e}}(z)=H_{\mathrm{s}}(z)+H_{\mathrm{a}}(z)$,

where

$H_{\mathrm{s}}(z)=\frac{1}{2}\left[H_{\mathrm{e}}(z)+z^{-(N-1)} H_{\mathrm{e}}\left(z^{-1}\right)\right]$,

$H_{\mathrm{a}}(z)=\frac{1}{2}\left[H_{\mathrm{e}}(z)-z^{-(N-1)} H_{\mathrm{e}}\left(z^{-1}\right)\right]$.

Note that $H_{\mathrm{s}}(z)$ represents a symmetric FIR filter and $H_{\mathrm{a}}(z)$ is an antisymmetric one. According to the above equations and Eq. (22), we have

$H_{\mathrm{o}}(z)=(-1)^{(N-1) / 2}\left[H_{\mathrm{s}}(z)-H_{\mathrm{a}}(z)\right]$.

Substituting Eq. (17) with Eqs. (23) and (26), the transfer function of proposed MF FHT is expressed as a linear combination of $H_{\mathrm{s}}(z)$ and $H_{\mathrm{a}}(z)$

$$
\begin{aligned}
H(z)= & \cos \phi\left[H_{\mathrm{s}}(z)+H_{\mathrm{a}}(z)\right] \\
& +(-1)^{(N-1) / 2} \sin \phi z^{-1}\left[H_{\mathrm{s}}(z)-H_{\mathrm{a}}(z)\right] .
\end{aligned}
$$

This structure is shown in Fig. 1.

Example 1. In this example, we demonstrate the symmetry of the impulse response discussed above

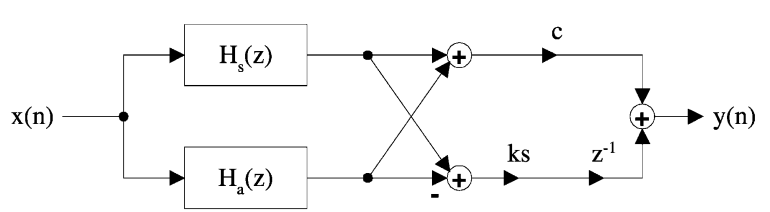

Fig. 1. Realization of the maximally flat fractional Hilbert transformers of odd order where $c=\cos (\alpha \pi / 2+N \pi / 4)$, $s=\sin (\alpha \pi / 2+N \pi / 4)$, and $k=(-1)^{(N-1) / 2} . N$ is an odd number. 
by exhibiting the nineth-order filter transfer function $H(z)$ explicitly. According to Eqs. (13)-(16), the impulse response of this nineth-order MF FHT FIR filter is

$$
\begin{aligned}
& h(0)=\frac{35 \cos \phi}{2048}, h(2)=\frac{63 \cos \phi}{512}, h(4)=\frac{945 \cos \phi}{1024}, \\
& h(6)=-\frac{105 \cos \phi}{512}, h(8)=-\frac{45 \cos \phi}{2048}, \\
& h(1)=-\frac{45 \sin \phi}{2048}, h(3)=-\frac{105 \sin \phi}{512}, \\
& h(5)=\frac{945 \sin \phi}{1024}, h(7)=\frac{63 \sin \phi}{512}, h(9)=\frac{35 \sin \phi}{2048} .
\end{aligned}
$$

Since $\hat{h}(2 n)=h(2 n) / \cos \phi$ and $\hat{h}(2 n+1)=h(2 n+1) /$ $\sin \phi, H_{\mathrm{e}}(z)$ and $H_{\mathrm{o}}(z)$ in Eq. (17) are calculated and expressed by

$$
\begin{aligned}
H_{\mathrm{e}}(z)= & \frac{35}{2048}+\frac{63}{512} z^{-2}+\frac{945}{1024} z^{-4}-\frac{105}{512} z^{-6} \\
& -\frac{45}{2048} z^{-8}
\end{aligned}
$$

and

$$
\begin{aligned}
H_{\mathrm{o}}(z)= & -\frac{45}{2048}-\frac{105}{512} z^{-2}+\frac{945}{1024} z^{-4} \\
& +\frac{63}{512} z^{-6}+\frac{35}{2048} z^{-8}
\end{aligned}
$$

respectively. Note the time-reversed symmetry between $H_{\mathrm{e}}(z)$ and $H_{\mathrm{o}}(z)$. According to Eqs. (24) and (25), $H_{\mathrm{e}}(z)$ and $H_{\mathrm{o}}(z)$ can be represented by $H_{\mathrm{e}}(z)=H_{\mathrm{s}}(z)+H_{\mathrm{a}}(z)$ and $H_{\mathrm{o}}(z)=H_{\mathrm{s}}(z)-H_{\mathrm{a}}(z)$ where

$$
\begin{aligned}
H_{\mathrm{s}}(z)= & -\frac{5}{2048}-\frac{21}{512} z^{-2}+\frac{945}{1024} z^{-4} \\
& -\frac{21}{512} z^{-6}-\frac{5}{2048} z^{-8}
\end{aligned}
$$

and

$$
H_{\mathrm{a}}(z)=\frac{5}{256}+\frac{21}{128} z^{-2}-\frac{21}{128} z^{-6}-\frac{5}{256} z^{-8} .
$$

$H_{\mathrm{s}}(z)$ are a symmetric transfer function and $H_{\mathrm{a}}(z)$ are an antisymmetric transfer function.

In [3], the authors proposed efficient algorithms to design fixed-point FIR filters. It can be shown that the impulse responses of odd order MF FHTs can be exactly expressed by fixed point binary values except the two irrational factors of $\cos \phi$ and $\sin \phi$ in Eq. (17). For example, $H_{\mathrm{e}}(z)$ can be expressed as

$$
\begin{aligned}
& H_{\mathrm{e}}(z)=\left(2^{-6}+2^{-10}+2^{-11}\right) \\
& \quad+\left(2^{-4}+2^{-5}+2^{-6}+2^{-7}+2^{-8}+2^{-9}\right) z^{-2} \\
& \quad+\left(2^{-1}+2^{-2}+2^{-3}+2^{-5}+2^{-6}+2^{-10}\right) z^{-4} \\
& \quad-\left(2^{-3}+2^{-4}+2^{-6}+2^{-9}\right) z^{-6} \\
& \quad-\left(2^{-6}+2^{-8}+2^{-9}+2^{-11}\right) z^{-8} .
\end{aligned}
$$

Since $H_{\mathrm{o}}(z)$ is the time-reversed version of $H_{\mathrm{e}}(z)$ and $H_{\mathrm{s}}(z)$ and $H_{\mathrm{a}}(z)$ are combinations of $H_{\mathrm{e}}(z)$ and $H_{\mathrm{o}}(z)$, they are able to be exactly expressed by fixed point binary numbers and implemented by the add and shift operations.

To evaluate the number of multiplications expressed by Eq. (27), note that the coefficients of the odd numbered impulse response are zeros in $H_{\mathrm{s}}(z)$ and $H_{\mathrm{a}}(z)$. Since they are linear phase FIR filters of $(N-1)$ th order, each of them has $(N+1) / 2$ nonzero coefficients and only halves are necessary for realization. We conclude that the number of multiplications for realizing the MF FHT shown in Fig. 1 is $(N+5) / 2$. This number is two more than that in the common linear phase FIR filter due to the factors $\sin \phi$ and $\cos \phi$.

\section{Design of even-order FIR FHTs}

In this section, we will solve the impulse responses of the even-order maximally flat FIR Hilbert transformers. For this case, the number of equations expressed by Eqs. (7) and (8) is examined. For the $k$ th derivative, two MF conditions must be satisfied since the MF frequencies are specified to be $\pi / 2$ and $-\pi / 2$. That is, there are two equations constructed for each choice of $k$. To solve the linear equations with $N+1$ unknowns expressed by Eqs. (7) and (8), $k$ is equal to $N / 2$ at most since $N$ is 
an even number. Hence there are $N$ linear equations constructed by the MF conditions. Because the number of the unknown impulse response is $N+1$, we must find another equation in order to solve $h(n)$. This additional equation is specified at the frequency of $\omega=0$ and expressed by $H\left(\mathrm{e}^{\mathrm{j} 0}\right)=$ $\left[H_{\mathrm{d}}\left(\mathrm{e}^{\mathrm{j} \pi / 2}\right)+H_{\mathrm{d}}\left(\mathrm{e}^{-\mathrm{j} \pi / 2}\right)\right] / 2$, i.e.,

$\sum_{n=0}^{N} h(n)=\cos (\alpha \pi / 2)$.

We will simplify Eqs. (7), (8) and (28) to solve them analytically. For each $k$, by adding and subtracting Eqs. (7) and (8), respectively, we deduce

$$
\begin{aligned}
& \sum_{n=0}^{N} h(n) n^{k} \cos \left(\frac{n \pi}{2}\right)=\left(\frac{N}{2}\right)^{k} \cos \phi, \\
& \sum_{n=0}^{N} h(n) n^{k} \sin \left(\frac{n \pi}{2}\right)=\left(\frac{N}{2}\right)^{k} \sin \phi
\end{aligned}
$$

for $k=0,1, \ldots, N / 2-1$. Since $\cos (n \pi / 2)=0$ and $\sin (n \pi / 2)=0$ for odd $n$ and even $n$ in each, the above equations are actually in the forms of

$$
\sum_{n=0}^{N / 2} h(2 n)(-1)^{n}(2 n)^{k}=\left(\frac{N}{2}\right)^{k} \cos \phi
$$

and

$$
\sum_{n=0}^{N / 2-1} h(2 n+1)(-1)^{n}(2 n+1)^{k}=\left(\frac{N}{2}\right)^{k} \sin \phi
$$

for $k=0,1, \ldots, N / 2-1$. Note that Eqs. (29)-(32) are similar to those of Eqs. (9)-(12) but the number of unknowns and the number of equations are different. Eq. (31) gives $N / 2$ linear equations with $(N / 2+1)$ unknowns $h(0), h(2), \ldots, h(N)$ and Eq. (32) gives $N / 2$ equations with $N / 2$ unknowns $h(1), h(3), \ldots, h(N-1)$. Therefore, we may obtain the impulse responses of odd index by solving Eq. (32). The even-indexed impulse response is solved from Eq. (31) as well as Eq. (28).

The solution to Eq. (32) are obtained by carrying out Cramer's rule and explicitly expressed by

$$
h(2 n+1)=\frac{\sin \phi \prod_{i=0}^{N / 2-1}[i-(N-2) / 4]}{[n-(N-2) / 4] n ![(N-2) / 2-n] !}
$$

for $n=0,1, \ldots, N / 2-1$. Since $h(2 n+1)$ is obtained, we are able to obtain the $N / 2+1$ evenindexed impulse response $h(2 n)$ by solving Eq. (31) together with Eq. (28). After some algebraic manipulations, these equations are solved analytically.

Case 1: $N=4 p-2$. In this case, the odd-indexed filter coefficients expressed by Eq. (33) are reduced to

$$
\begin{aligned}
& h(2 p-1)=\cos (\alpha \pi / 2), \\
& h(2 n+1)=0 \quad \text { for } n=0,1, \ldots, 2 p-2, n \neq p-1 .
\end{aligned}
$$

Substituting Eq. (34) for Eqs. (31) and (28), the even-indexed impulse response is explicitly solved as

$$
\begin{aligned}
& h(2 n)=\frac{\sin (\alpha \pi / 2) \prod_{i=0}^{p-1}(i+1 / 2)^{2}}{(n-p+1 / 2) n !(2 p-1-n) !} \\
& \quad \text { for } n=0,1, \ldots, 2 p-1 .
\end{aligned}
$$

Case 2: $N=4 p$. If the filter order $N$ is a multiple of 4, the odd-indexed filter coefficients in Eq. (33) are simplified and expressed as

$$
\begin{aligned}
& h(2 n+1)=\frac{\sin (\alpha \pi / 2) \prod_{i=0}^{p-1}(i+1 / 2)^{2}}{(n-p+1 / 2) n !(2 p-1-n) !} \\
& \text { for } n=0,1, \ldots, 2 p-1 .
\end{aligned}
$$

Substituting Eq. (36) for Eqs. (31) and (28), the even-indexed impulse response is explicitly solved as

$$
\begin{aligned}
& h(2 p)=\cos (\alpha \pi / 2), \\
& h(2 n)=0 \text { for } n=0,1, \ldots, 2 p, n \neq p .
\end{aligned}
$$

There is an interesting relation between the MF FHT of orders $4 p-2$ and $4 p$. Let $h(n)$ be the filter coefficients of order $4 p-2$ and $h^{\prime}(n)$ be those of order $4 p$. According to Eq. (37), $h^{\prime}(0)=h^{\prime}(4 p)=0$. Comparing Eq. (34) with Eq. (37), it is obvious that $h(2 n+1)=h^{\prime}(2 n)=0 \quad$ and $\quad h(2 n)=h^{\prime}(2 n+1)$. These properties indicate that the MF FHT of order $4 p$ is equivalent to the MF FHT of order $4 p-2$ except an additional delay. In other word, for the design of the even-order FIR MF FHTs, it is sufficient to design the FHTs of order $4 p-2$. 
Example 2. The impulse responses of the filters of 10th and 12th orders are listed as follows.

\begin{tabular}{|c|c|c|c|c|c|c|c|c|c|c|c|c|}
\hline$n$ & 0 & 1 & 2 & 3 & 4 & 5 & 6 & 7 & 8 & 9 & 10 & 11 \\
\hline$N=10$ & $-3 s^{\prime}$ & 0 & $-25 s^{\prime}$ & 0 & $-150 s^{\prime}$ & $256 c^{\prime}$ & $150 s^{\prime}$ & 0 & $25 s^{\prime}$ & 0 & $3 s^{\prime}$ & \\
\hline$N=12$ & 0 & $-3 s^{\prime}$ & 0 & $-25 s^{\prime}$ & 0 & $-150 s^{\prime}$ & $256 c^{\prime}$ & $150 s^{\prime}$ & 0 & $25 s^{\prime}$ & 0 & $3 s^{\prime}$ \\
\hline
\end{tabular}

It is obvious for the impulse responses that the 13 coefficients of $N=12$ is the delayed version of $N=10$. Note that $c^{\prime}=\cos (\alpha \pi / 2), s^{\prime}=\sin (\alpha \pi / 2)$, and all the coefficients are multiplied by 256 to obtain integers. Then the integral coefficients of the even order MF FHTs can be implemented by fixed point operation without any multiplication.

Eq. (34) shows the symmetry of the coefficients. Since $h(2 n)=-h(N-2 n)$ and the odd-indexed coefficients are equal to zero except the central coefficient $h(N / 2)$, the frequency response can be written in the following way:

$$
H\left(\mathrm{e}^{\mathrm{j} \omega}\right)=\mathrm{e}^{\mathrm{j} N \omega / 2}\left[\cos (\alpha \pi / 2)+\sin (\alpha \pi / 2) \tilde{H}\left(\mathrm{e}^{\mathrm{j} \omega}\right)\right] .
$$

On the other hand, the desired frequency response $H_{\mathrm{d}}\left(\mathrm{e}^{\mathrm{j} \omega}\right)$ given in Eq. (2) can be expressed as

$$
H_{\mathrm{d}}\left(\mathrm{e}^{\mathrm{j} \omega}\right)=\mathrm{e}^{\mathrm{j} N \omega / 2}[\cos (\alpha \pi / 2) \pm \mathrm{j} \sin (\alpha \pi / 2)],
$$

where " + " is taken for $\omega<0$ and " - " for $\omega>0$. Equating $H\left(\mathrm{e}^{\mathrm{j} \omega}\right)$ with $H_{\mathrm{d}}\left(\mathrm{e}^{\mathrm{j} \omega}\right)$, we conclude that

$$
\tilde{H}\left(\mathrm{e}^{\mathrm{j} \omega}\right)=\left\{\begin{array}{rc}
-\mathrm{j} & \text { for } \omega>0, \\
\mathrm{j} & \text { for } \omega<0
\end{array}\right.
$$

\section{Table 1}

A summary of the impulse responses of the maximally flat fractional Hilbert transformers with transfer function $H(z)=\sum_{n=0}^{N} h(n) z^{-n}$

\begin{tabular}{ll}
\hline Order & Impulse response \\
\hline$N=$ & $h(N / 2)=\cos (\alpha \pi / 2)$ \\
$4 p-2$ & $h(2 n+1)=0, n=0,1, \ldots, 2 p-2$ and $n \neq p-1$ \\
& $h(2 n)=\frac{\sin (\alpha \pi / 2) \prod \prod_{i=0}^{p-1}(i+1 / 2)^{2}}{(n-p+1 / 2) n !(2 p-1-n) !}$, \\
& $n=0,1, \ldots, 2 p-1$ \\
$N=4 p$ & $h(N / 2)=\cos (\alpha \pi / 2)$ \\
& $h(2 n)=0, n=0,1, \ldots, 2 p$ and $n \neq p$ \\
& $h(2 n+1)=\frac{\sin (\alpha \pi / 2) \prod \prod_{i=0}^{p-1}(i+1 / 2)^{2}}{(n-p+1 / 2) n !(2 p-1-n) !}$, \\
& $n=0,1, \ldots, 2 p-1$ \\
$N=$ & $h(2 n)=\frac{\cos (\alpha \pi / 2+N \pi / 4) \prod{ }_{i=0}^{q}(i-N / 4)}{(n-N / 4)(q-n) ! n !}$, \\
$2 q+1$ & $n=0,1, \ldots, q$ \\
& $h(2 n+1)=\frac{\sin (\alpha \pi / 2+N \pi / 4) \prod{ }_{i=0}^{q}(i-N / 4+1 / 2)}{(n-N / 4+1 / 2)(q-n) ! n !}$, \\
& $n=0,1, \ldots, q$
\end{tabular}

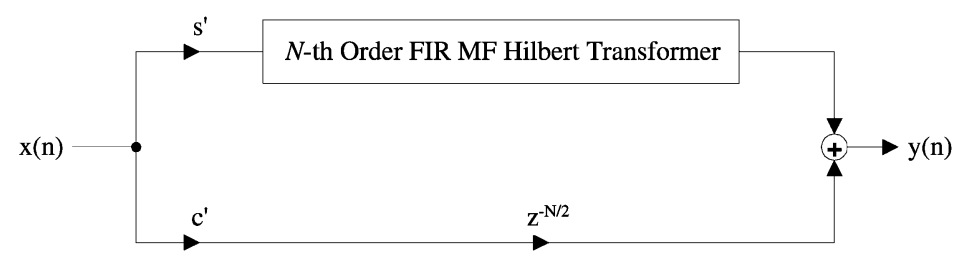

Fig. 2. Realization of the maximally flat fractional Hilbert transformers of even order. Note that $s^{\prime}=\sin (\alpha \pi / 2), c^{\prime}=\cos (\alpha \pi / 2)$. 


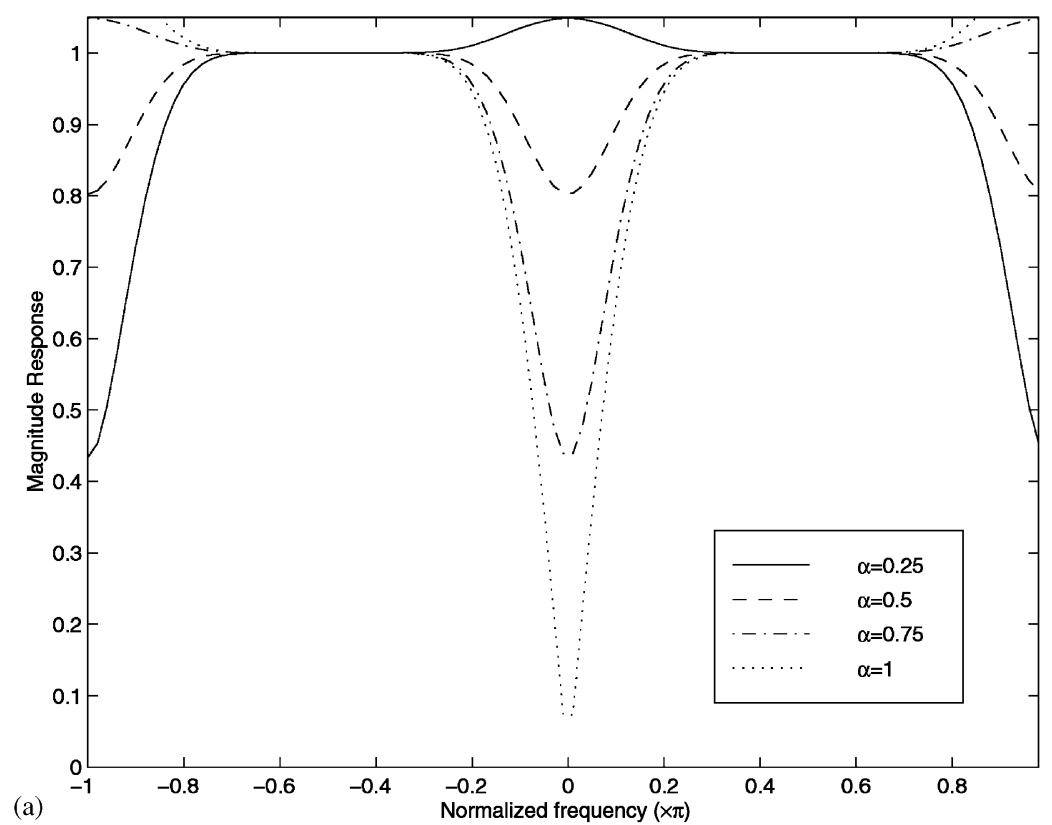

(a)

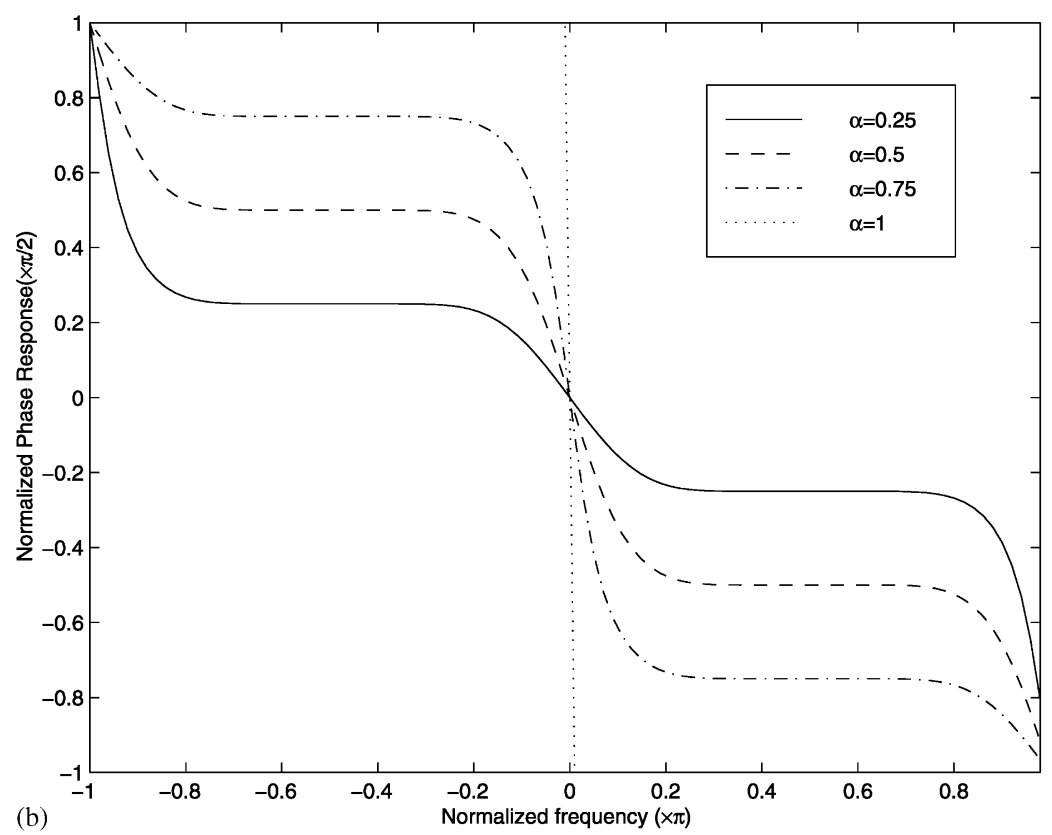

Fig. 3. Frequency responses of 17th-order FIR MF FHT with various values of $\alpha=0.25,0.5,0.75$, and 1.0: (a) magnitude responses, (b) phase responses. The linear component of the phase response is removed.

That is, $\tilde{H}\left(\mathrm{e}^{\mathrm{j} \omega}\right)$ is just the Hilbert transformer. Based on the above discussion, we conclude that the fractional Hilbert transformer of even order can be realized by adding an extra path and two multipliers of $\cos (\alpha \pi / 2)$ and $\sin (\alpha \pi / 2)$ to the $\mathrm{MF}$ Hilbert transformer [1]. This realization structure 


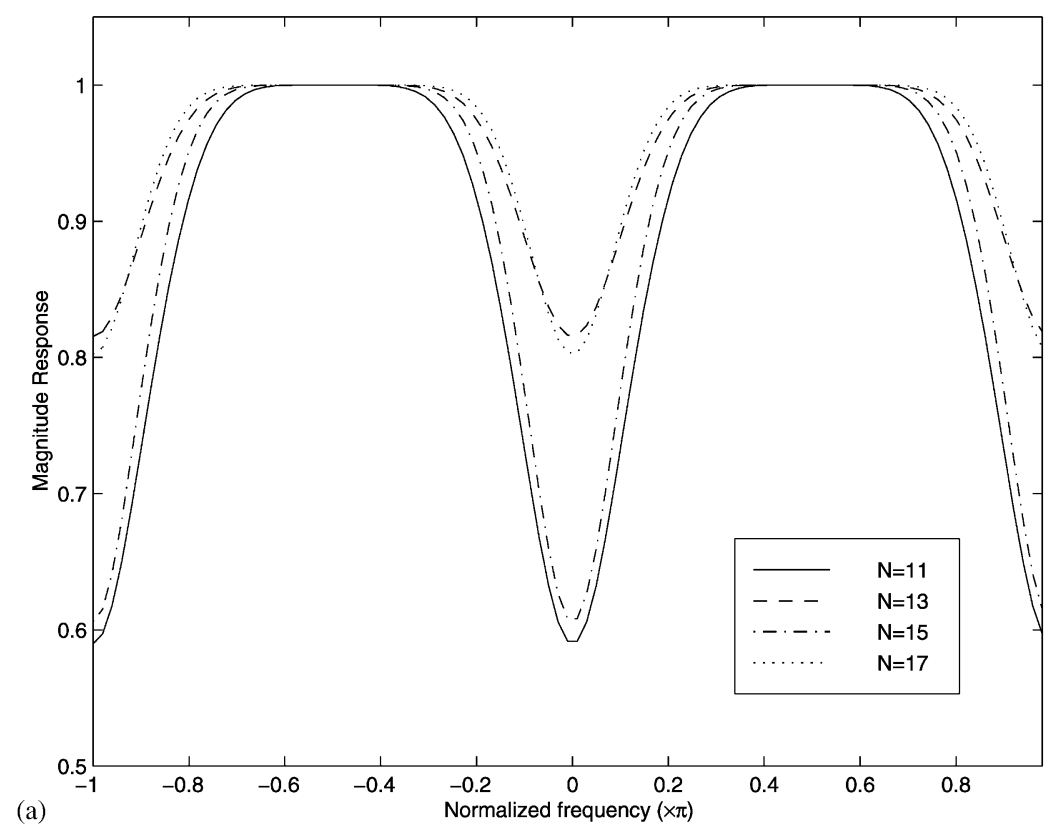

(a)

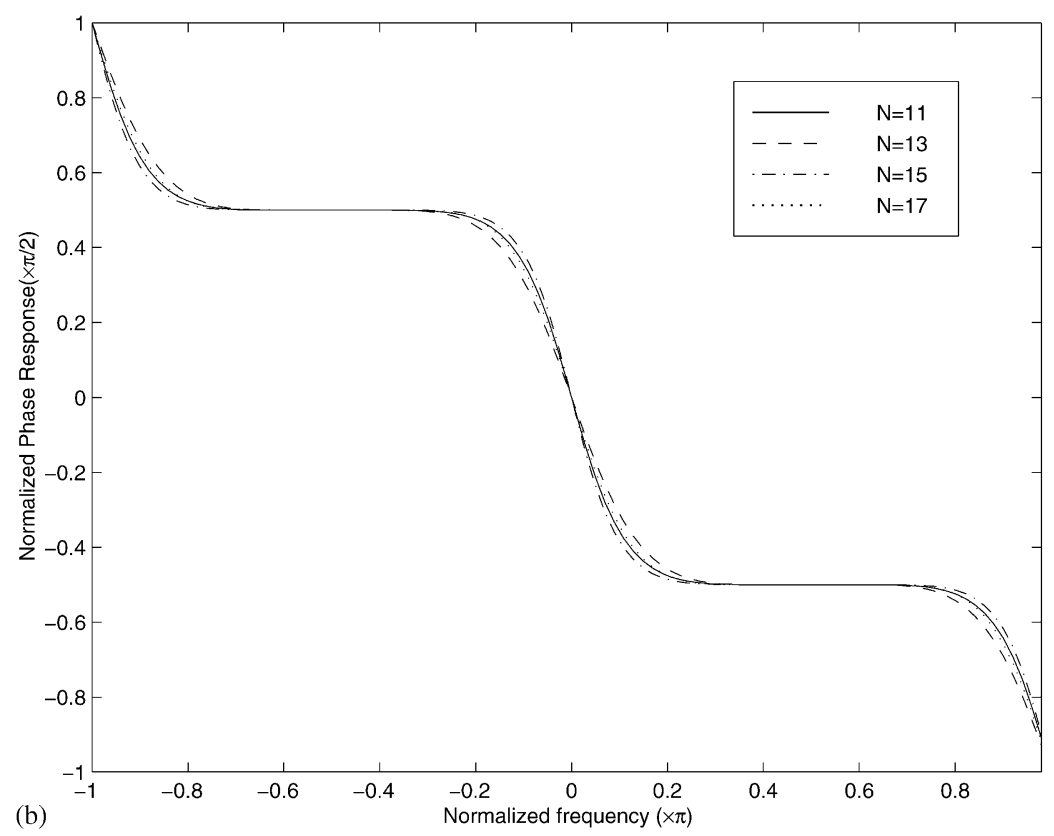

Fig. 4. Frequency responses of FIR MF FHT of various filter orders 10, 14, 18, and 22 with $\alpha=0.5$ : (a) magnitude responses, (b) phase responses.

of the fractional Hilbert transformer shown is in Fig. 2. Table 1 gives a summary of the impulse responses.

\section{Design examples}

In this section, we demonstrate some examples of the proposed maximally flat fractional Hilbert 

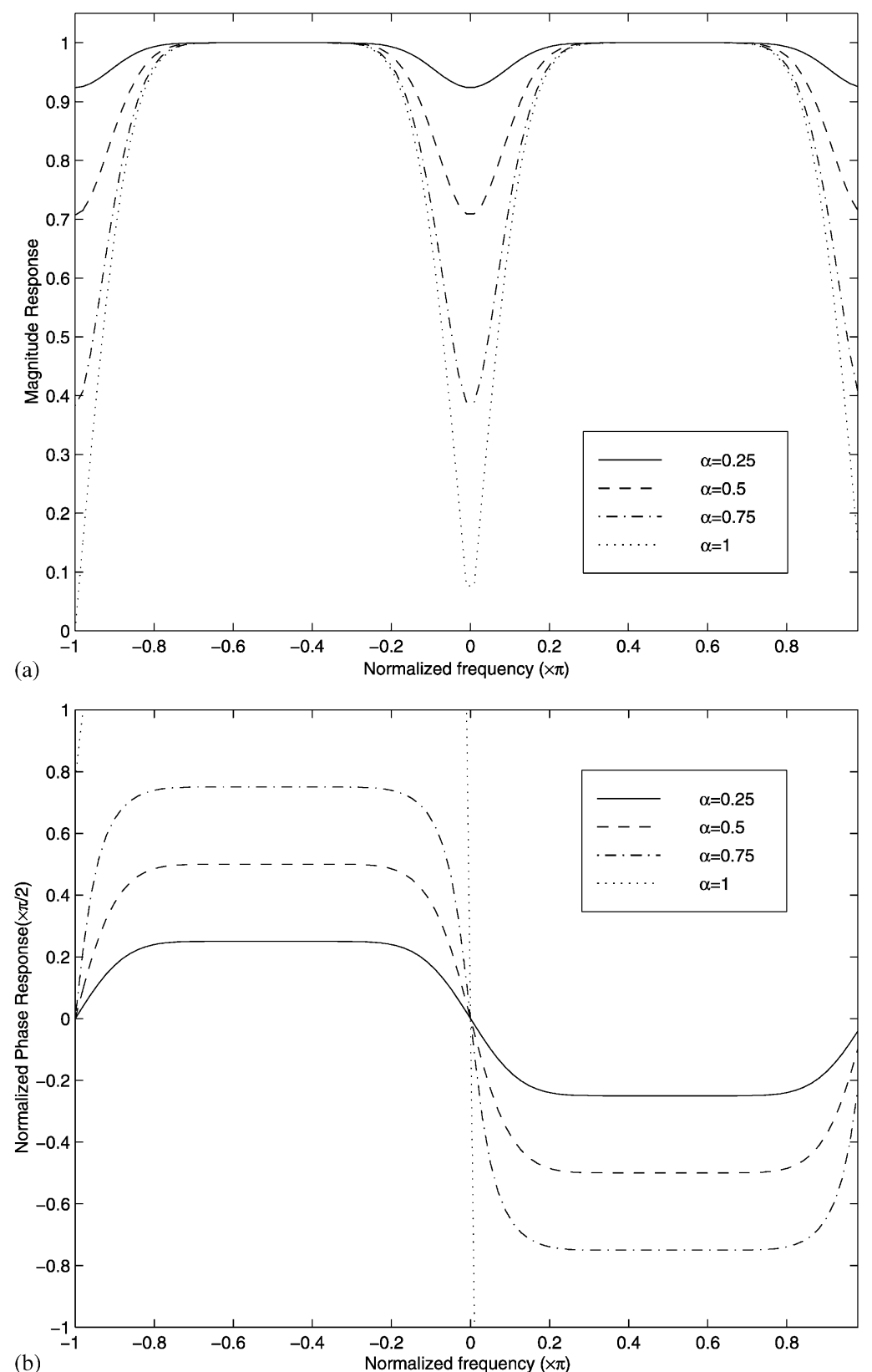

Fig. 5. Frequency responses of 18 th-order FIR MF FHT with various values of $\alpha=0.25,0.5,0.75$, and 1.0: (a) magnitude responses, (b) phase responses.

transformers for various parameters and orders. The filter is applied to a rectangle function. This can be regarded as an example of the 1-D edge detection.
Example 3. In this example, a set of maximally flat fractional Hilbert transformers of 17th order are designed. The fractional factors $\alpha$ are $0.25,0.5,0.75$, and 1 for each filter. Figs. $3 a$ and $b$ show the 


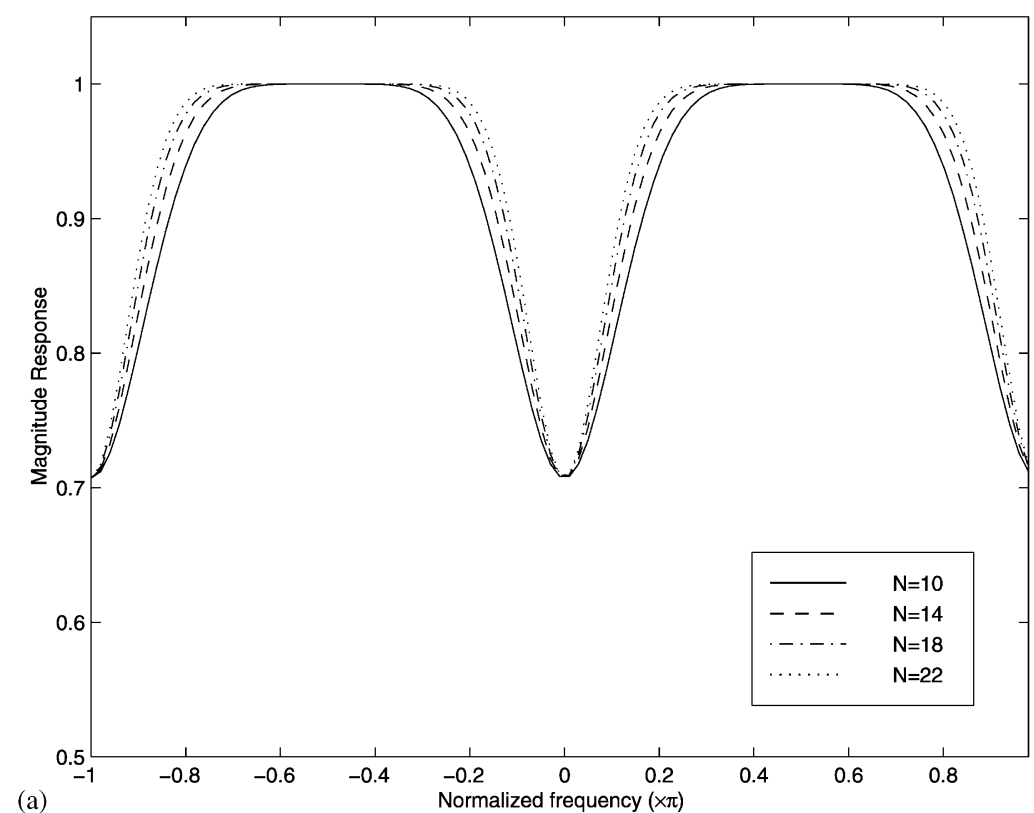

(a)

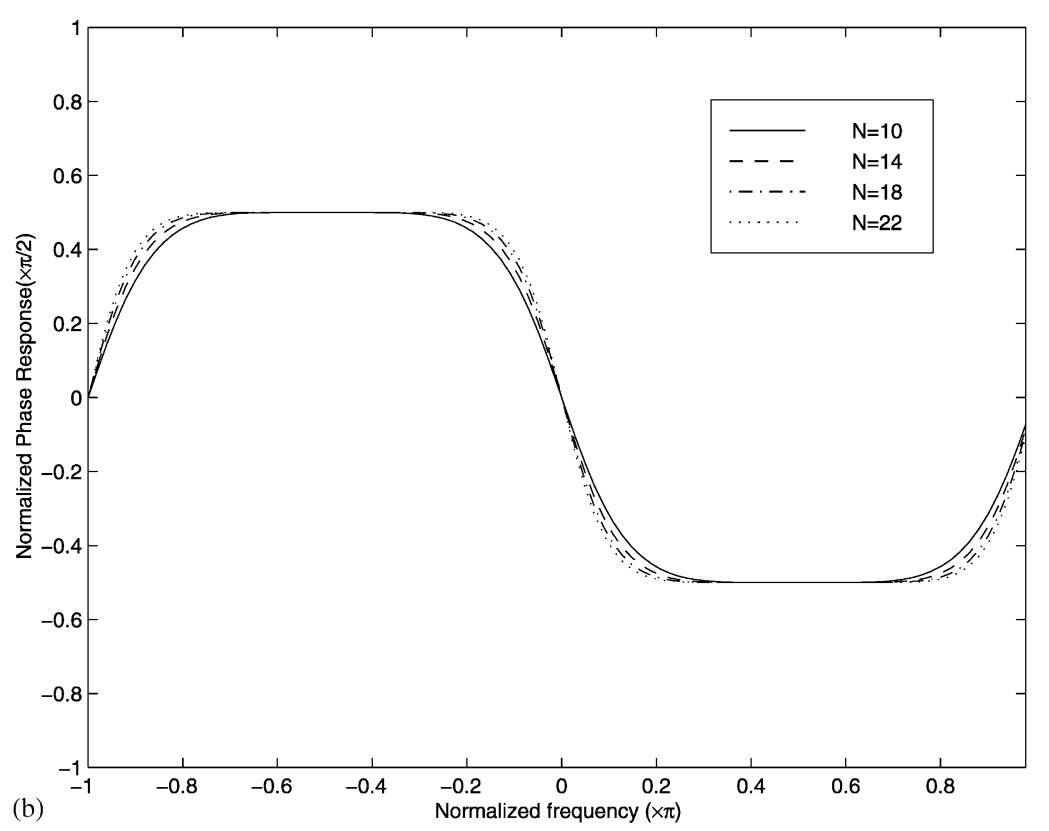

Fig. 6. Frequency responses of FIR MF FHT of various orders with $\alpha=0.5$ : (a) magnitude responses, (b) phase responses.

magnitude and the phase responses, respectively. The linear component of the phase responses is removed in the following manner:

$\tilde{\theta}(\omega)=\theta(\omega)+\frac{N \omega}{2}$ where $\tilde{\theta}(\omega)$ and $\theta(\omega)$ are the modified phase response and the actual phase response, respectively. $N$ is the filter order and equal to 17 in this example. There is a bump of the magnitude response around $\omega=0$ for $\alpha=0.25$. For $\alpha>0.5$, bumps occur around $\omega=\pi$. 


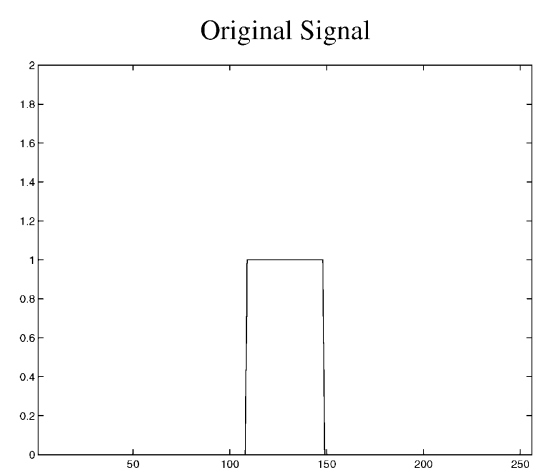

$\alpha=0.5$

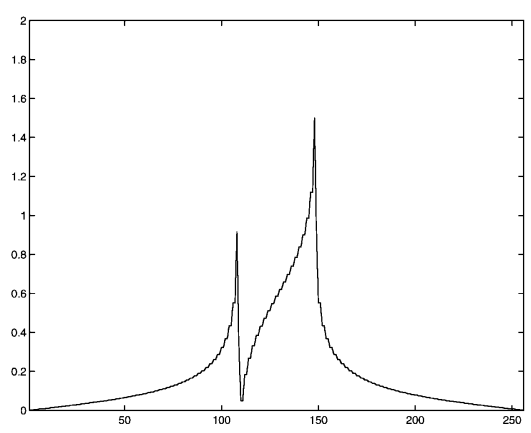

$\alpha=1.25$

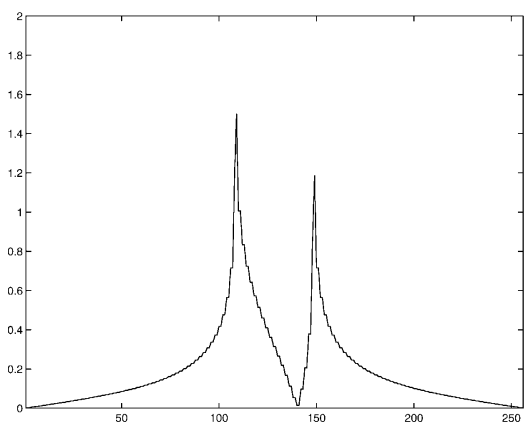

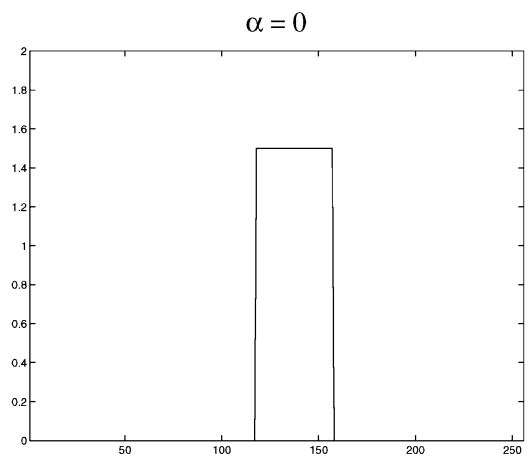

$\alpha=0.75$

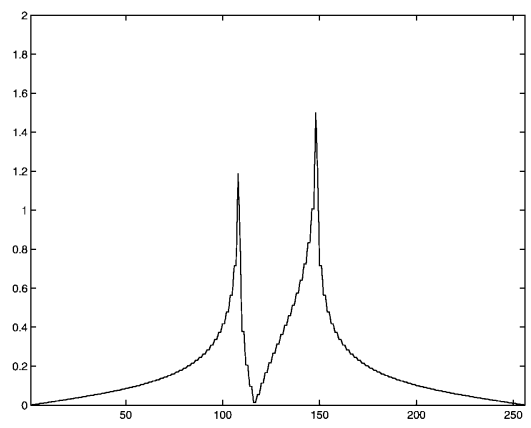

$\alpha=1.5$

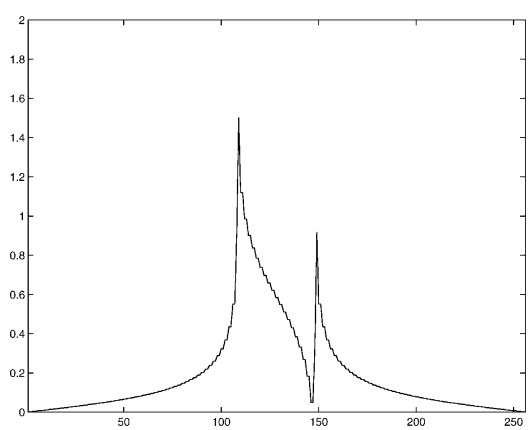

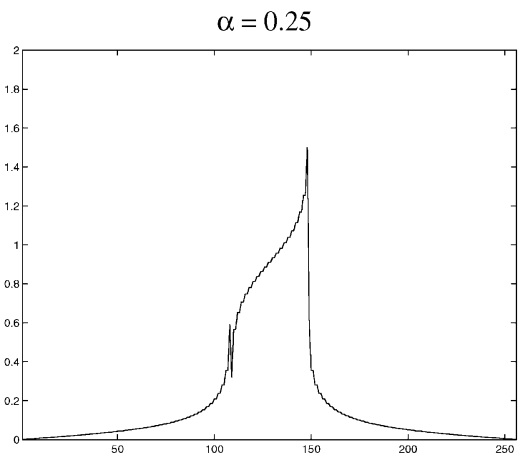

$\alpha=1$

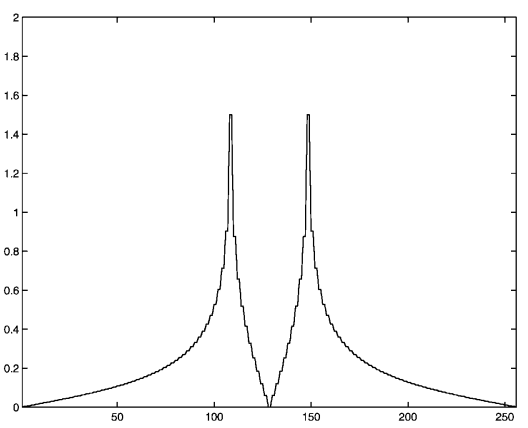

$\alpha=1.75$

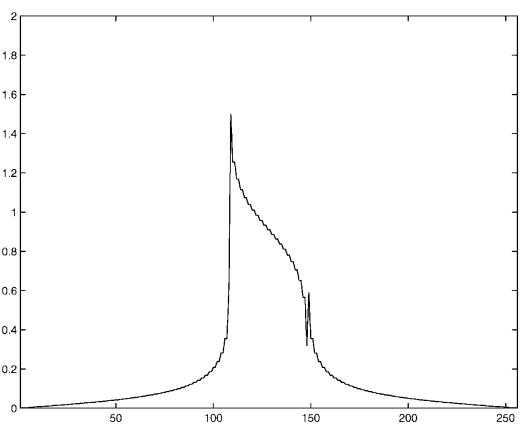

Fig. 7. Fractional Hilbert transform of a discrete rectangular function performed by discrete-time Fourier transform proposed in [13] for various values of $\alpha$. The results are normalized to obtain maximal output levels of 1.5 .

Example 4. The filters of different orders are designed with $\alpha=0.5$ in this example. The orders are 11, 13, 15, and 17. Fig. 4a shows the magnitude responses and Fig. $4 \mathrm{~b}$ shows the normalized phase responses. The bandwidth is increased if the filter order is increased. Note that the curves of magnitude divide into two groups. The magnitude response of $N=11$ is close to that of $N=15$. Similarity occurs for $N=13$ and 17. However, all the phase responses are similar.

Example 5. In this example, a set of maximally flat fractional Hilbert transformers of 18th order are designed. The fractional factors $\alpha$ are $0.25,0.5,0.75$, and 1 for each filter. Figs. 5a and $\mathrm{b}$ show the 

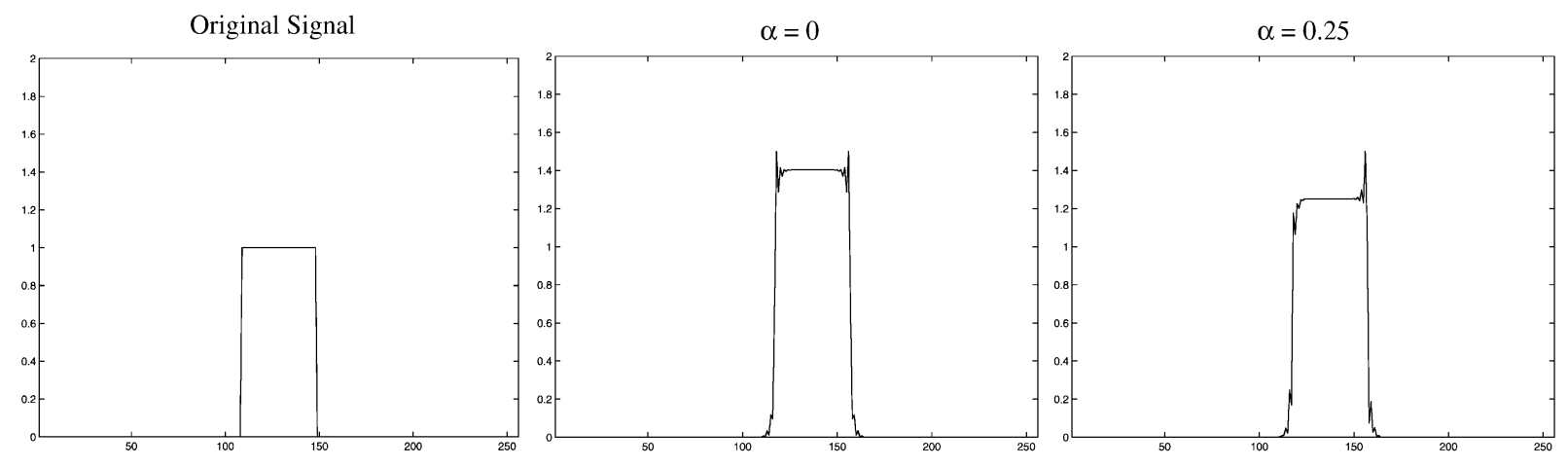

$\alpha=0.5$

$\alpha=0.75$
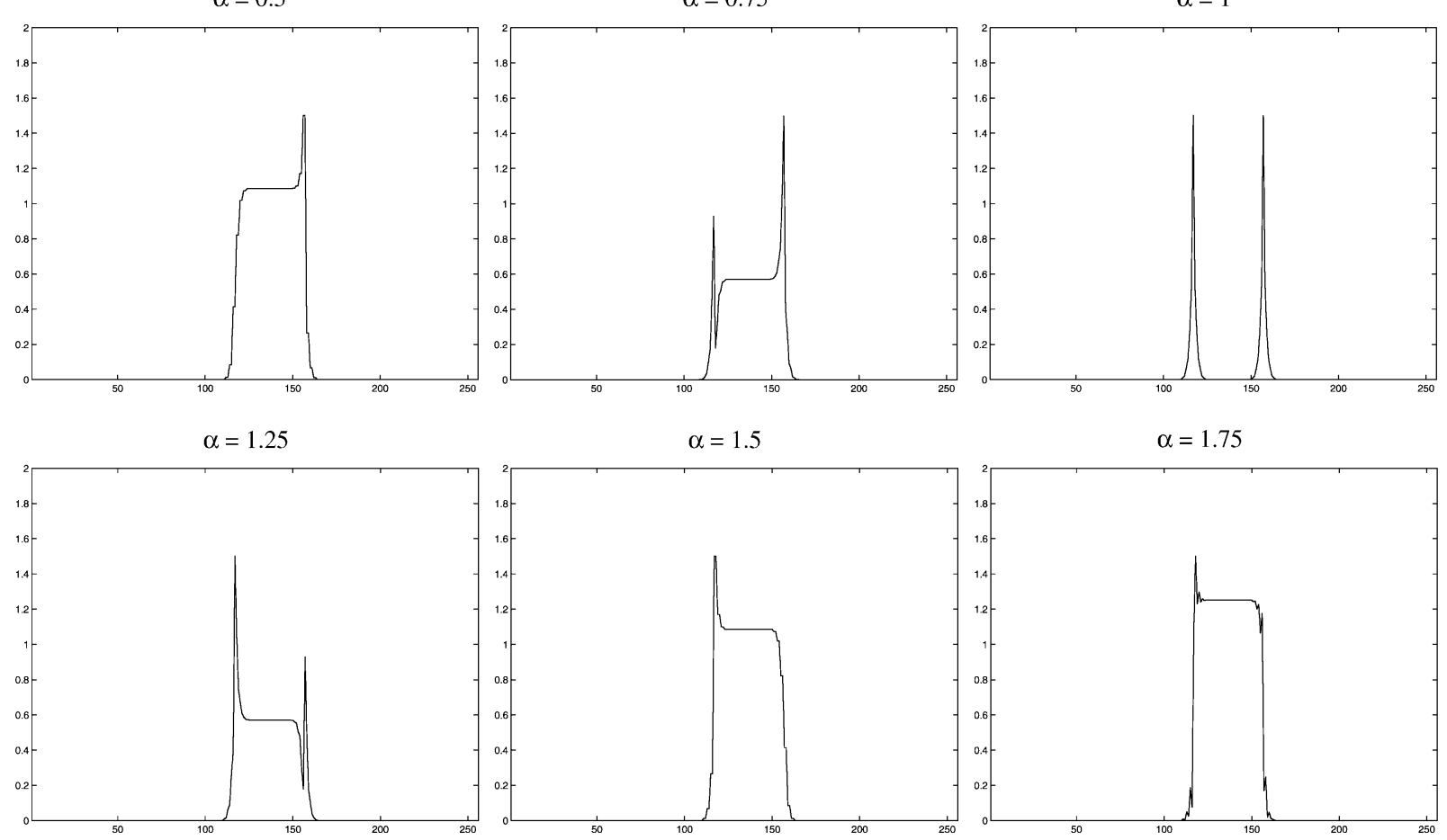

Fig. 8. Fractional Hilbert transform of a discrete rectangular function performed by MF FIR filter of 17 th order for various values of $\alpha$. The results are normalized to obtain maximal output levels of 1.5 .

magnitude and phase responses, respectively. There is no bump on the magnitude response.

Example 6. The filters of different orders are designed with $\alpha=0.5$ in this example. The orders are 10, 14, 18, and 22. Fig. 6a shows the magnitude responses and Fig. 6b shows the normalized phase responses. The bandwidth is increased if the filter order is increased. Unlike the frequency responses of the odd-order MF FHTs, there is no obvious gap for the magnitude curves.

Example 7. In this example, the proposed MF FHTs are used to detect the edges for 1-D signals. Both even- and odd-order filters are used. This test signal is a discrete rectangular function given by

$x(n)= \begin{cases}1 & \text { for } 108 \leqslant n \leqslant 147, \\ 0 & \text { for } 0 \leqslant n \leqslant 107 \text { or } 148 \leqslant n \leqslant 255 .\end{cases}$ 

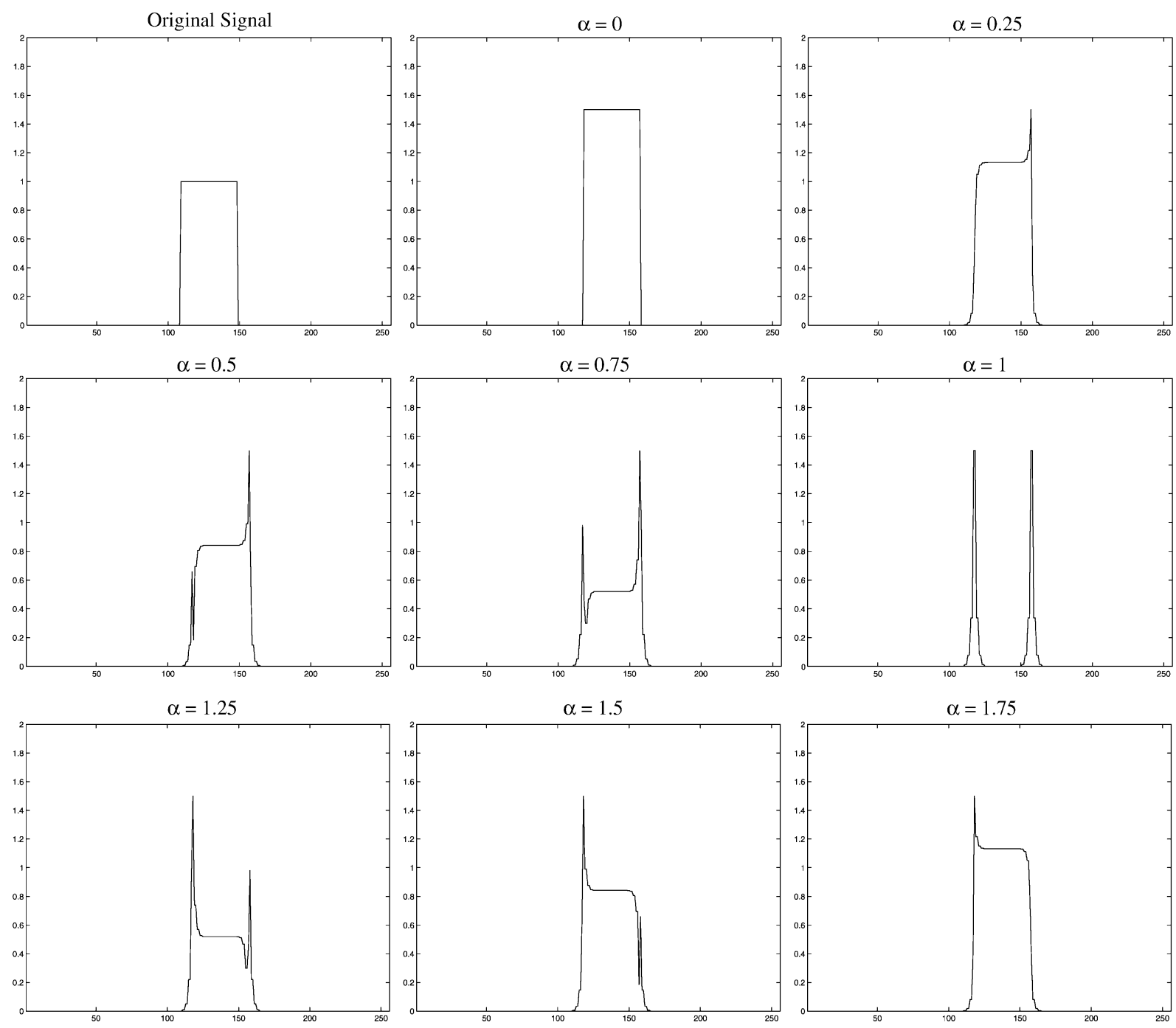

Fig. 9. Fractional Hilbert transform of a discrete rectangle function performed by MF FIR filter of 18 th order for various values of $\alpha$. The results are normalized to obtain maximal output levels of 1.5 .

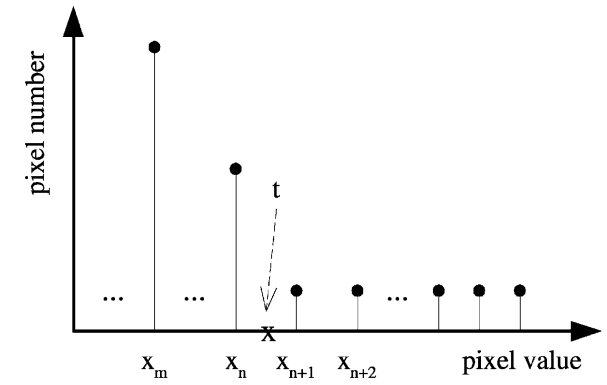

Fig. 10. Choice of the threshold for the 2-D edge detection. $x_{n}$ is the pixel value with the second largest pixel number. The threshold is computed by $\left(x_{n}+x_{n+1}\right) / 2$.
That is, the width of $x(n)$ is equal to 40 points and its length is 256 points. This signal is also used for demonstration in $[8,13]$. Fig. 7 shows the magnitude responses of the fractional Hilbert transform of the test signal where the transform is performed based on the discrete-time Fourier transform proposed in [13].

Fig. 8 shows the magnitude responses of the filtering outputs when the test signal is filtered by the MF FHT of the 17 th order. In comparison with the transform result shown in Fig. 7, it is obvious that the outputs obtained by 

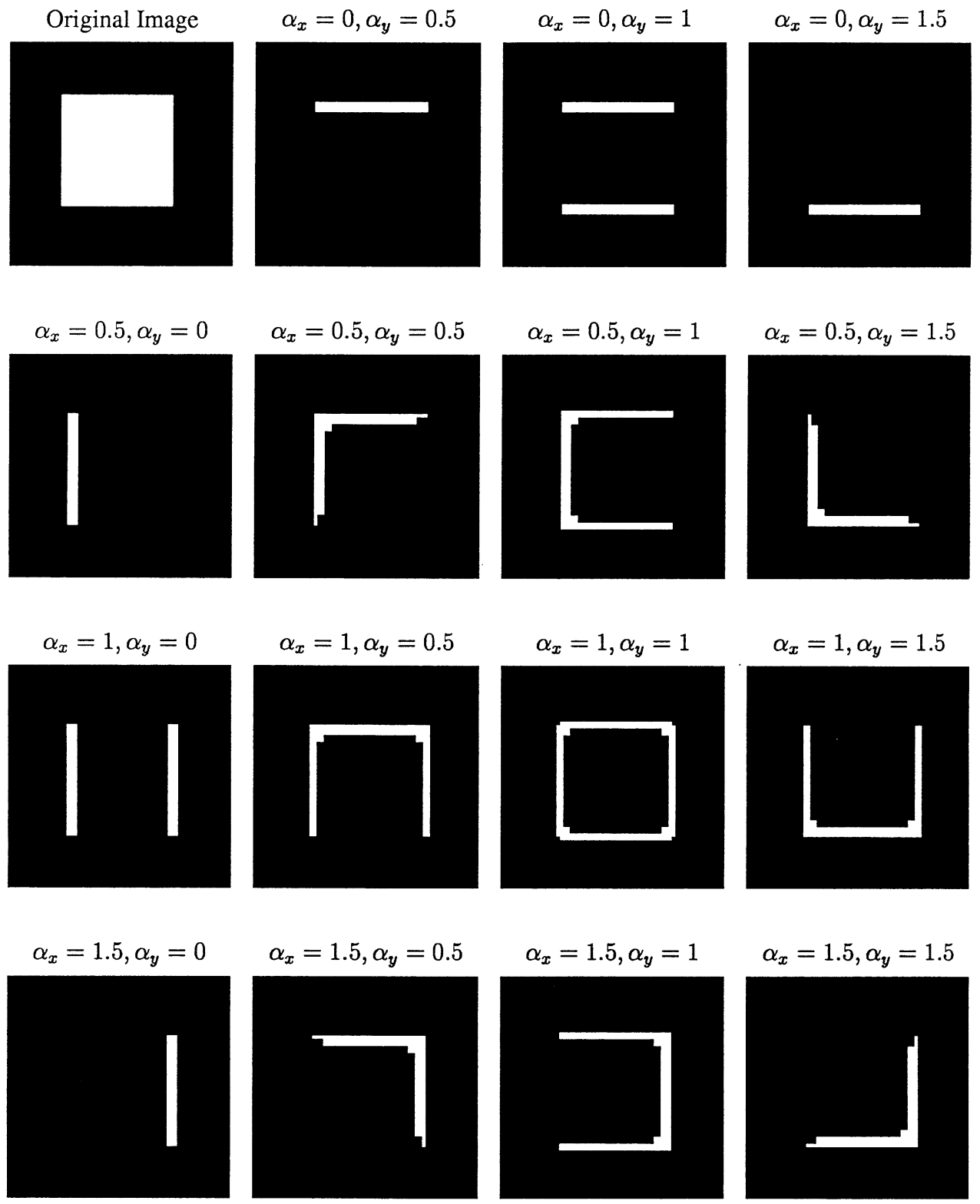

Fig. 11. Fractional Hilbert transform of a 2-D rectangle function. The test image is filtered by the proposed MF FHT of 10th order for various values of $\alpha_{x}$ and $\alpha_{y}$.

the proposed FIR filter decay faster than the outputs of the test image. That is to say, the filter output may detect the edges more precisely. In Fig. 9 the magnitude responses of the filtering outputs are shown if the test signal is filtered by the MF FHT of the 18th order. The rising edges are emphasized for $0<\alpha<1$, whereas the falling edges are emphasized for $1<\alpha<2$. If $\alpha=1$, both types of the edges are equally emphasized. Note that the overshoots occur at the edges for $\alpha=0$ in Fig. 8. This inconsistency is overcome if the filter order is even. 

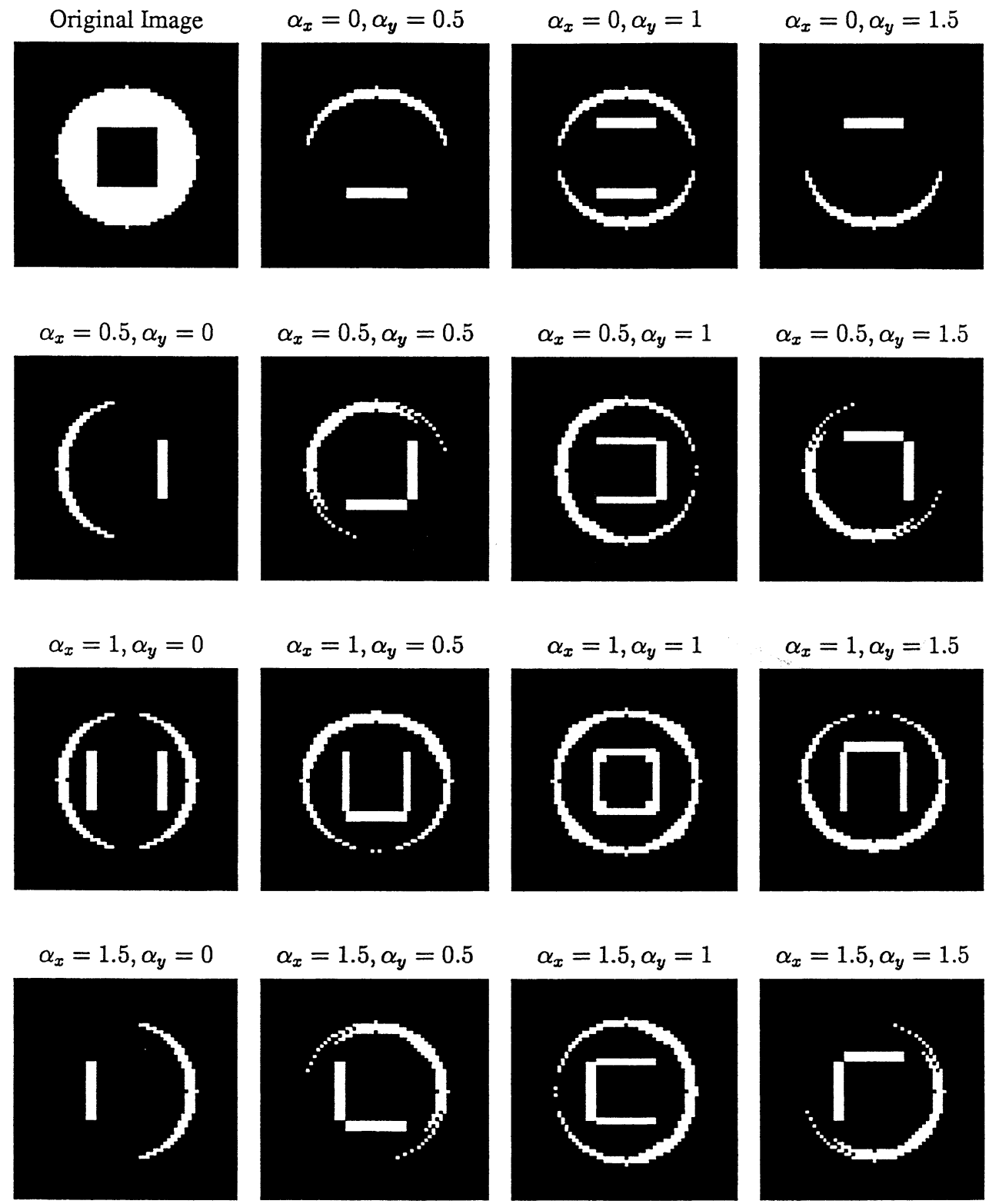

Fig. 12. Fractional Hilbert transform of a digital image consisted of a circular region with a rectangle hole. The test image is filtered by the proposed MF FHT of 10th order for various values of $\alpha_{x}$ and $\alpha_{y}$.

Example 8. In this example, we apply the proposed FIR maximally flat Hilbert transformer to detect the edges for the digital images. The 10th-order filter with different $\alpha$ is used for filtering. The edges are detected at the $(m, n)$ point if the following condition is satisfied

$\left|h_{\alpha_{x}}(m, n)\right|^{2}+\left|h_{\alpha_{y}}(m, n)\right|^{2}>t$,

where $h_{\alpha_{x}}(m, n)$ and $h_{\alpha_{y}}(m, n)$ are the outputs of the FIR FHT with parameter $\alpha_{x}$ and in the horizontal 

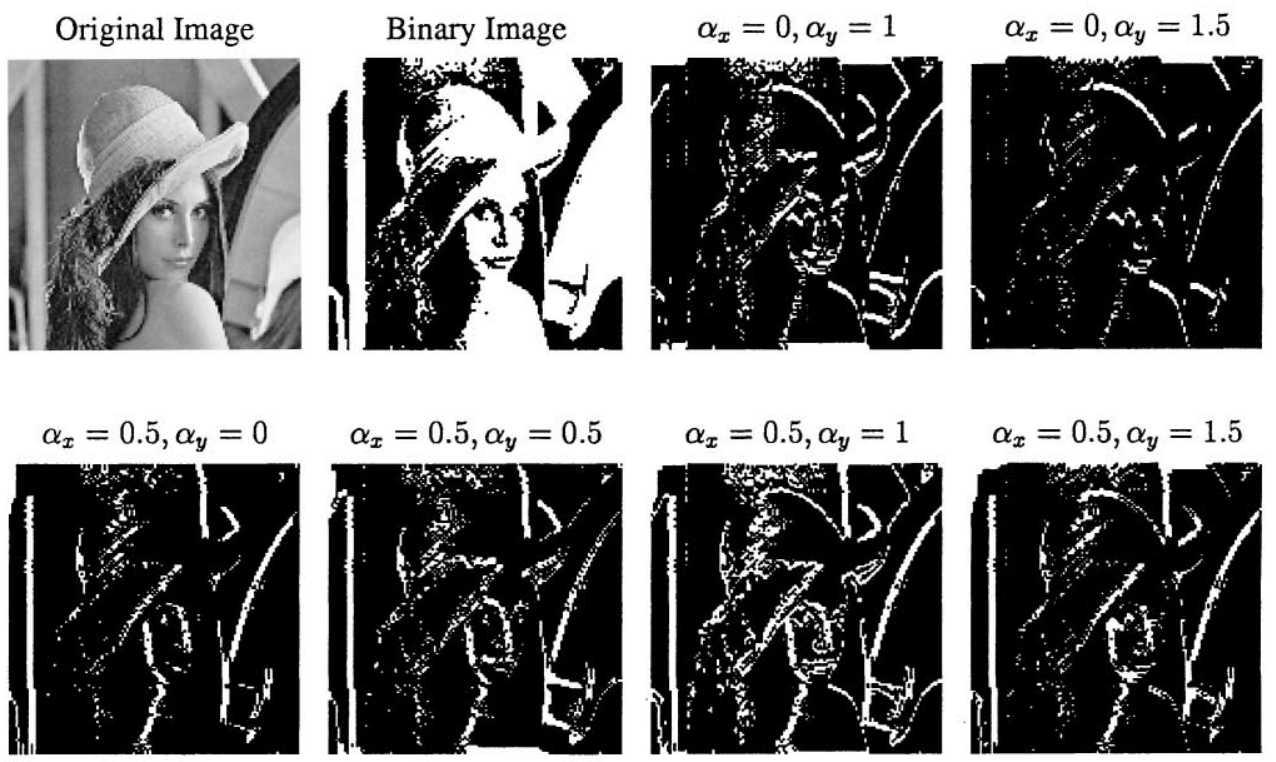

$\alpha_{x}=1, \alpha_{y}=0$

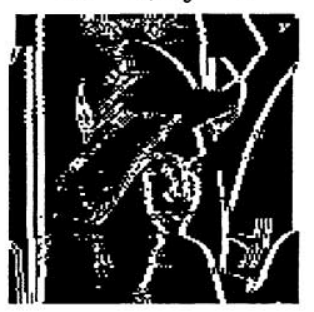

$\alpha_{x}=1.5, \alpha_{y}=0$

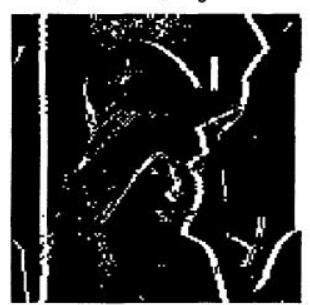

$\alpha_{x}=1, \alpha_{y}=0.5$

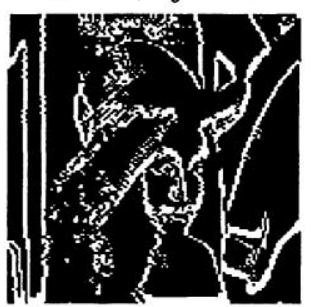

$\alpha_{x}=1.5, \alpha_{y}=0.5$

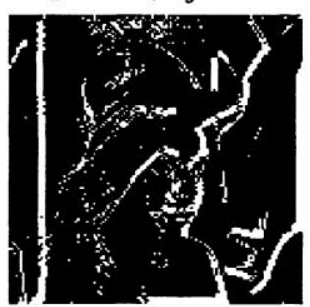

$\alpha_{x}=1, \alpha_{y}=1$

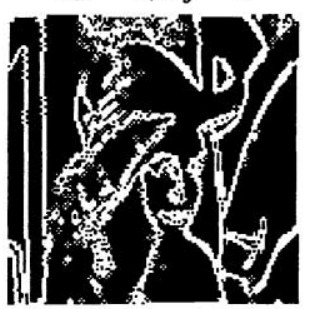

$\alpha_{x}=1.5, \alpha_{y}=1$

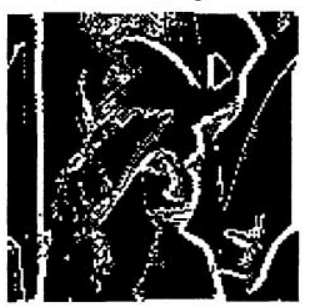

$\alpha_{x}=1 ; \alpha_{y}=1.5$

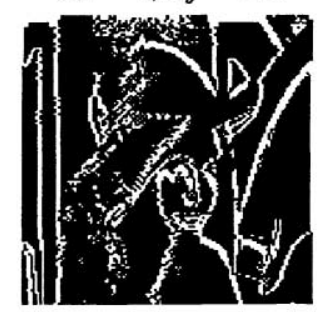

$\alpha_{x}=1.5, \alpha_{y}=1.5$

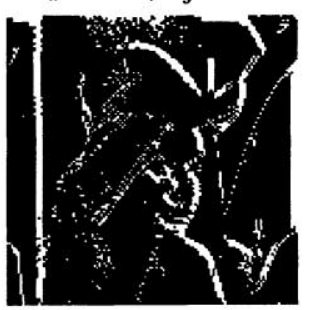

Fig. 13. Fractional Hilbert transform of the digital image "Lena". The original image is binary threshold and then filtered by the proposed MF FHT of 10th order for various combinations of $\alpha_{x}$ and $\alpha_{y}$.

direction and $\alpha_{y}$ in the vertical direction, respectively. The computation of the threshold $t$ which is based on the histogram of $\left|h_{\alpha_{x}}(m, n)\right|^{2}$ $+\left|h_{\alpha_{v}}(m, n)\right|^{2}$. Suppose $x_{n}$ is the pixel value with the second largest pixel number, the threshold is computed by

$t=\frac{x_{n}+x_{n+1}}{2}$.

This choice of $t$ is demonstrated by Fig. 10 . 
Fig. 11 shows the results of a test image filtered by different $\alpha_{x}$ and $\alpha_{y}$. The foreground of the test image is a rectangle. Like the conditions discussed in Example 7, the rising edges in the horizontal direction are detected for $0<\alpha_{x}<1$. The falling edges in the horizontal direction are emphasized for $1<\alpha_{x}<2$. Both edges are detected if $\alpha_{x}=1$. The edges in the vertical can be selectively detected while suitable $\alpha_{y}$ are chosen.

Fig. 12 shows the detected edges in another digital image. The test image is a circular region with a rectangular hole. Both the rising edges and the falling edges are precisely located.

For edge detection of a natural images, we choose the image "Lena" for demonstration. Fig. 13 shows the original image together with the filtered images. The original image is a binary threshold by the moment-preserving thresholding [17] and normalized between 0 and 1 before filtering by the proposed MF FHT of the 10th order. For different choice of $\alpha_{x}$ and $\alpha_{y}$, different edges are detected.

\section{Conclusions}

In this paper, the design of the maximally flat FIR fractional Hilbert transformers is proposed. The impulse responses are solved analytically for both even- and odd-order filter. Although the impulse responses of the MF FHTs cannot be expressed in fixed-point binary number exactly, the MF FHT can be implemented efficiently where the coefficients are simple rational numbers and can be expressed in sum of power-of-two based on the discussions and examples in Sections 3 and 4. Therefore, the filter coefficients can be implemented by add and shift operations after suitable scaling. Two multiplications are needed at most. Although the impulse responses of the $(4 p-2)$ th and $4 p$ th order are derived separately, the analytical results indicate that these two cases are actually identical.
The $4 p$ th-order FIR maximally flat fractional Hilbert transformers are delayed versions of the $(4 p-2)$ th-order ones. Based on Eqs. (38) and (39), the even-order MF FIR FHTs can be synthesized by classical Hilbert transformers.

Efficient hardware realization structures are obtained for the maximally flat fractional Hilbert transformers. The structures of the odd-order filters are derived based on symmetric properties of the coefficients. This structure reduces half of the multipliers necessary for implementation. The even-order fractional Hilbert transformers are realized by putting one extra path and two multipliers on the classical Hilbert transformers.

\section{Appendix}

Derivation of Eqs. (13), (14) and (33): According to the equivalent matrix form of Eq. (13), the solution can be expressed by

$\tilde{h}(2 n)=\operatorname{det} \boldsymbol{A}_{n} / \operatorname{det} \boldsymbol{A}, \quad n=0,1, \ldots,(N-1) / 2$

based on Cramer's rule [10] where

$$
\boldsymbol{A}=\left(\begin{array}{ccccc}
1 & 1 & 1 & \cdots & 1 \\
0 & 2 & 4 & \cdots & (N-1) \\
0 & 2^{2} & 4^{2} & \cdots & (N-1)^{2} \\
\vdots & \vdots & \vdots & \ddots & \vdots \\
0 & 2^{(N-1) / 2} & 4^{(N-1) / 2} & \cdots & (N-1)^{(N-1) / 2}
\end{array}\right)
$$

and $\boldsymbol{A}_{n}$ are formed by replacing the $n$th column of $\boldsymbol{A}$ with the column vector of

$$
\left(1, N / 2,(N / 2)^{2}, \ldots,(N / 2)^{(N-1) / 2}\right)^{\mathrm{T}} \text {. }
$$

Since both of $\operatorname{det} \boldsymbol{A}$ and $\operatorname{det} \boldsymbol{A}_{n}$ are Vandermonde determinants [17], it is well known that $\operatorname{det} \boldsymbol{A}=\prod_{i<j}\left(z_{j}-z_{i}\right)$ and $\operatorname{det} \boldsymbol{A}_{n}=\prod_{i<j}\left(z_{j}^{\prime}-z_{i}^{\prime}\right)$, where $z_{i}=2 i$ for $i=0,1, \ldots,(N-1) / 2, z_{i}^{\prime}=2 i$ for $i=0,1, \ldots, n-1, n+1, \ldots,(N-1) / 2, \quad$ and $z_{n}^{\prime}=$ $N / 2$. Therefore, we obtain

$$
\begin{aligned}
\tilde{h}(2 n) & =\frac{(N / 2-0)(N / 2-2) \times \cdots \times[N / 2-(2 n-2)][(2 n+2)-N / 2] \times \cdots \times(N-1-N / 2)}{(2 n-0)(2 n-2) \times \cdots \times[2 n-(2 n-2)][(2 n+2)-2 n] \times \cdots \times(N-1-2 n)} \\
& =\frac{(-1)^{n} \prod_{i=0}^{(N-1) / 2}(2 i-N / 2)}{2^{(N-1) / 2}(2 n-N / 2)((N-1) / 2-n) ! n !}=\frac{(-1)^{n} \prod_{i=0}^{(N-1) / 2}(i-N / 4)}{(n-N / 4)((N-1) / 2-n) ! n !}
\end{aligned}
$$


and

$$
\begin{aligned}
h(2 n) & =\tilde{h}(2 n)(-1)^{n} \cos \phi \\
& =\frac{k_{\mathrm{e}} \cos \phi}{(n-N / 4)((N-1) / 2-n) ! n !},
\end{aligned}
$$

where $k_{\mathrm{e}}$ is expressed in Eq. (15).

Based on the similar formulation and manipulation, Eqs. (14) and (33) are obtained.

\section{References}

[1] T. Cooklev, A. Nishihara, Maximally flat FIR Hilbert transformer, Int. J. Circuit Theory Appl. 21 (1993) 353-570.

[2] J.A. Davis, D.E. McNamara, Don M. Cottrell, Analysis of the fractional Hilbert transform, Appl. Opt. 37 (29) (October 1998) 6911-6913.

[3] A.G. Dempster, M.D. Macleod, Use of minimum-adder multiplier blocks in FIR digital filters, IEEE Trans. Circuits Systems - II 42 (9) (September 1995) 569-577.

[4] G.H. Golub, C.F. Van Loan, Matrix Computation, The Johns Hopkins University Press, Baltimore, MD, 1989.

[5] H. Johansson, L. Wanhammar, Digital Hilbert transformers composed of identical allpass subfilters, Proceedings of the IEEE International Symposium on Circuits and Systems, Monterey, CA, May 1998.

[6] K. Kohlmann, Corner detection in natural images based on the 2-D Hilbert transform, Signal Processing 48 (1996) 225-234.

[7] J. Le Bihan, Coefficients of FIR digital differentiators and Hilbert transformers for midband frequencies, IEEE Trans. Circuits Systems - II 43 (3) (March 1996) 272-274.
[8] A.W. Lohmann, D. Mendlovic, Z. Zalevsky, Fractional Hilbert transform, Opt. Lett. 21 (4) (February 1996) 281-283.

[9] J.H. McClellan, T.W. Parks, L.R. Rabiner, A computer program for designing optimum FIR linear phase digital filters, IEEE Trans. Audio Electroacoust. AU-21 (December 1973) 506-526.

[10] B. Noble, J.W. Daniel, Applied Linear Algebra, PrenticeHall, Englewood Cliffs, NJ, 1988.

[11] A.V. Oppemheim, R.W. Schafer, Discrete-Time Signal Processing, Prentice-Hall, Englewood Cliffs, NJ, 1989.

[12] S.-C. Pei, P.-H. Wang, Analytical design of digital nonrecursive maximally flat fractional Hilbert transformer, Proceedings of the IEEE International Symposium on Circuits and Systems, Orlando, FL, May 1999.

[13] S.-C. Pei, M.-H. Yeh, Discrete fractional Hilbert transform, Proceedings of the IEEE International Symposium on Circuits and Systems, Monterey, CA, May 1998.

[14] S.-C. Pei, M.-H. Yeh, C.-C. Tseng, Discrete fractional Fourier transform based on orthogonal projections, IEEE Trans. Signal Process. 47 (5) (May 1999) 1335-1348.

[15] L.R. Rabiner, B. Gold, Theory and Application of Digital Signal Processing, Prentice-Hall, Inc, Englewood Cliffs, NJ, 1975.

[16] L.R. Rabiner, J.H. McClellan, T.W. Parks, FIR digital filter disign techniques using weighted Chebyshev approximation, Proc. IEEE 63 (April 1975) 595-610.

[17] W.-H. Tsai, Moment-preserving thresholding: a new approach, Comp. Vision Graphics Image Process. 29 (1985) 377-393.

[18] Y.-L. Tai, T.-P. Lin, Design of Hilbert transformers by multiple use of same subfilter, Electron. Lett. 25 (19) (September 1989) 1288-1290. 